\title{
Influence of Different Turbulence Models on Flow Field Simulation of Nozzle Adjustable Jet Pump
}

\author{
ZHAO Xu-He $\mathrm{H}^{1,2, \mathrm{a}}$, YAN Ting-Jun ${ }^{1,2, \mathrm{~b}}$ and WANG Xiao-He $\mathrm{e}^{1,2, \mathrm{c}}$ \\ 1State Key Laboratory of Offshore Oil Exploitation, China \\ 2College of Mechanical and Electrical Engineering, Beijing University of Chemical Technology, \\ China \\ axuhe0112@163.com, byantj555@163.com, cturbo 21c@163.com
}

\begin{abstract}
Keywords: Adjustable jet pump; Turbulence model; Wall function; Numerical simulation
\end{abstract}
Abstract: In order to find a turbulent flow model which can accurately describe the internal mixing process of the nozzle adjustable jet pump, 8 combinations made up by four turbulence models (Standard $k-\varepsilon$, RNG $k-\varepsilon$, Realizable $k-\varepsilon$ and RSM) and two wall functions (Standard wall functions and Non-Equilibrium wall functions) were simulated and analyzed. Taking a nozzle adjustable jet pump as an example, the simulation of 8 models were carried out when the opening of the jet pump at 50\%,60\%,70\%,75\% and 90\%, respectively. And then the field tests were carried out. The comparison between the simulation results and the experimental data of the pressure ratio and efficiency curve was studied. And the turbulent kinetic energy distribution of the jet pump in different models was analyzed. The results show that: the difference of the wall function has little effect on the simulation results, the difference of the curve is mainly caused by differences in turbulence models. The error between the simulated value and the test value of each model decreases with the increase of opening degree and pressure ratio. The simulation results of the RNG $k-\varepsilon$ model have larger deviation compare with the experimental results, therefore it's not suitable for the simulation of the jet pump. The simulation results of Standard $k-\varepsilon$ and RSM model are similar, but exist errors. Realizable $k-\varepsilon$ model can predict the internal flow of the nozzle jet pump well, and it is in good agreement with the experimental data. The Non-Equilibrium wall function is more suitable for the simulation of jet flow, which is closer to the experimental value than the standard wall function. Therefore, the accurate performance prediction and flow field details can be obtained by the combination of Realizable $k-\varepsilon$ model and non-equilibrium wall function.

\section{Introduction}

The jet pump is a kind of fluid machinery, which uses jet diffusion ${ }^{[1]}$, and has been widely concerned in the field of petrochemical industry ${ }^{[2]}$. The working principle of the jet pump is that the high-pressure fluid flow through the conical nozzle, the speed was increased, causing pressure drop near the nozzle, the low-pressure fluid is sucked into the suction chamber under the pressure difference, mixed with the high-pressure working fluid, exchange energy and momentum in the throat, eventually pressurized out in the diffusion tube ${ }^{[3]}$. Because of its nozzle fixed, the ordinary jet pump's outlet flow pressure variable range is small, that is difficult to meet the actual needs. The biggest advantage of the nozzle adjustable jet pump is that a spray needle nozzle at the center of the nozzle, it can adjust the opening of the needle, so as to meet the different outlet pressure and flow rate of the actual demand ${ }^{[4,5]}$. The motion of the internal fluid in a nozzle adjustable jet pump is turbulent jet flow in an irregular finite area. It's very complex and brings difficulties to the simulation, and the simulation results of different turbulence models are different to the actual flow field in varying degrees. Therefore, it is necessary to compare and analyze the differences between different models and select the turbulence model which can accurately describe the characteristics of adjustable jet pump $^{[6,7]}$. In this paper, four turbulent models, Standard $k-\varepsilon$ (SKE), RNG $k-\varepsilon$ (RNGKE), Realizable $k-\varepsilon$ (RKE) and RSM, compared with two kinds of wall functions, Standard wall functions(SWF) and Non-Equilibrium wall functions(NWF), were designed to simulate the internal flow field of the 
nozzle adjustable jet pump. Through comparison and analysis, the optimum model combination of the internal flow field simulation of the adjustable jet pump is obtained.

\section{Test}

\section{Determined the size of the jet pump}

The main structure of adjustable jet pump is composed of nozzle, spray needle, throat, diffusion pipe and the suction chamber, etc. The main performance parameters of nozzle adjustable nozzle jet pump are flow ratio $q$, pressure ratio $h$, efficiency $\eta$ and nozzle opening $S r^{[8]}$. The definitions are as follow:

$$
\begin{aligned}
& q=\frac{Q_{s}}{Q_{o}} . \\
& h=\frac{P_{c}-P_{s}}{P_{o}-P_{s}} . \\
& \eta=h \cdot q . \\
& S_{r}=\frac{A_{o i}}{A_{o}} .
\end{aligned}
$$

where $Q_{s}$ is intake water flows, and $Q_{o}$ is power fluid flows $\left(\mathrm{m}^{3} / \mathrm{d}\right) ; P_{C}, P_{S}$ and $P_{O}$ represent the pressure of the mixed liquid, intake liquid and the power fluid respectively(MPa); $A_{o i}$ means actual flow area of nozzle, $A_{o}$ means overflow area of the nozzle without injection needle $\left(\mathrm{mm}^{2}\right)$.

This article is based on the actual demand of water injection pump on an offshore platform in Bohai sea. The design target is maintained the export pressure is $1.4 \sim 4.0 \mathrm{MPa}$, and the export flow is $500 \sim 800 \mathrm{~m}^{3} / \mathrm{d}$, providing working liquid $10 \mathrm{MPa}$, and the intake fluid is $1 \mathrm{MPa}$. Considering the constraints of the sea platform space and other factors ${ }^{[9]}$, a nozzle adjustable jet pump was calculated and designed ${ }^{[10]}$, whose basic structure is shown in Fig.1, and the size is shown in Table 1.

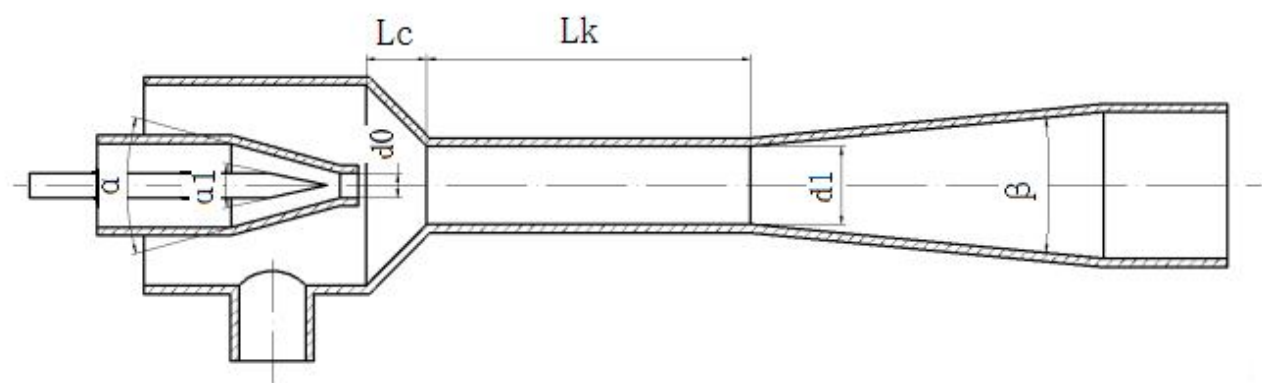

Fig.1 Key dimensions of jet pump

Table 1 Main structure dimensions of jet pump

\begin{tabular}{cccccccc}
\hline $\begin{array}{c}\text { Structure } \\
\text { name }\end{array}$ & $\begin{array}{c}\text { nozzle } \\
\text { diameter } \\
\mathrm{do} /[\mathrm{mm}]\end{array}$ & $\begin{array}{c}\text { angle of } \\
\text { nozzle } \\
\alpha /\left[^{\circ}\right]\end{array}$ & $\begin{array}{c}\text { throat } \\
\text { diameter } \\
\mathrm{d} /[\mathrm{mm}]\end{array}$ & $\begin{array}{c}\text { throat } \\
\text { length } \\
\mathrm{L}_{\mathrm{k}} /[\mathrm{mm}]\end{array}$ & $\begin{array}{c}\text { angle of } \\
\text { diffusion } \\
\text { tube } \beta /\left[^{\circ}\right]\end{array}$ & $\begin{array}{c}\text { angle of } \\
\text { needle } \\
\alpha_{1} /\left[{ }^{\circ}\right]\end{array}$ & $\begin{array}{c}\text { nozzle-to- } \\
\text { throat } \\
\mathrm{Lc} /[\mathrm{mm}]\end{array}$ \\
\hline $\begin{array}{c}\text { Design } \\
\text { results }\end{array}$ & 8 & 20 & $\Phi 13$ & 98 & 8 & 20 & 19.5 \\
\hline
\end{tabular}

\section{Test data acquisition}

After the manufacture has been processed, the test would be carried out. The test flow is shown in Fig. 2. During the test, the pressure of the working fluid and the intake fluid were stabilitated, the nozzle 
opening was fixed at $50 \%$, changed the pressure ratio by adjusting the valve V3 and recorded the flowmeter reading when the monitor was stability. After this experiment was completed, the equipment should be restored. Then adjusted the nozzle opening at $60 \%, 70 \%, 75 \%$ and $90 \%$ respectively. Repeat the above steps and recorded the test data as shown in Table 2.

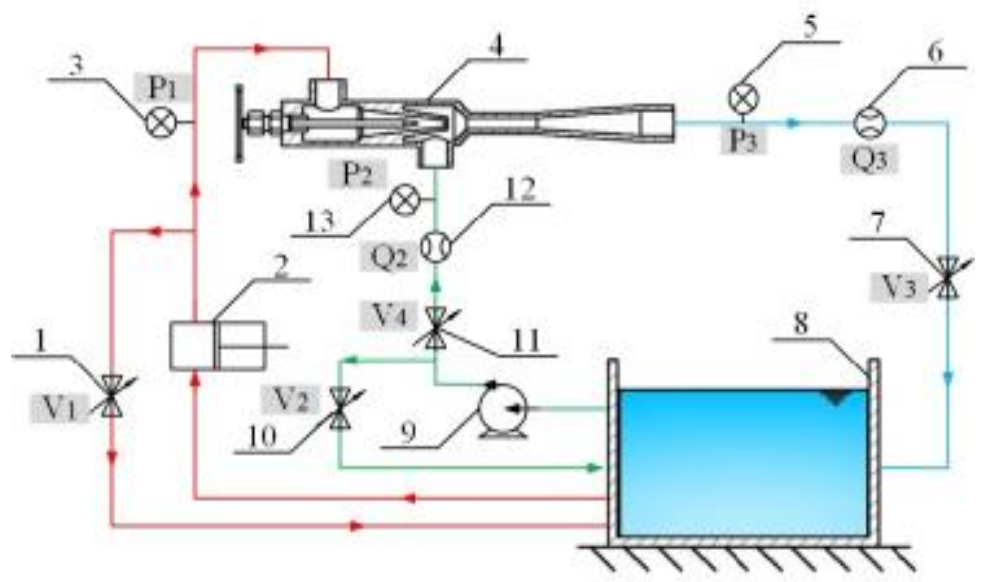

1, 7 valve; 2 high-pressure pump; 3,5,12 pressure gauge and pressure sensor; 4 water injection adjustable jet pump; 6,11 flowmeter; 8 pool; 9 frequency transformer; 10 centrifugal pump;

Fig. 2 Test flow chart

Table 2. Test of outlet flow $\left[\mathrm{m}^{3} / \mathrm{d}\right]$

\begin{tabular}{cccccc}
\hline \multirow{2}{*}{$\begin{array}{c}\text { Outlet pressure } \\
\text { /[MPa] }\end{array}$} & $50 \%$ & $60 \%$ & $70 \%$ & $75 \%$ & $90 \%$ \\
\cline { 2 - 6 } & 605 & 641 & 696 & 734 & 803 \\
\hline 1.4 & 529 & 568 & 635 & 678 & 792 \\
2.0 & 401 & 447 & 533 & 585 & 755 \\
3.0 & 273 & 325 & 432 & 491 & 657 \\
\hline .0 & & & & & \\
\hline
\end{tabular}

\section{CFD validation}

\section{CFD model and Settings}

In this paper, four turbulence models(SKE, RNGKE, RKE, RSM) and two kinds of wall functions (SWF, NWF) were used to simulate. In the simulation, the internal flow of the jet pump was simplified to incompressible flow and the heat exchange was neglected. The boundary conditions were pressure inlet and pressure outlet, and the pressure-velocity coupling adopted SIMPLEC algorithm, the spatial discretization adopted the Second Order Windward Format. All models adopted unstructured grids. In order to validate the grid-independent, took opening 50\% as an example, used 1.26 times to adjust the global size scale factor, generated 800,000, 1.5 million, and 2.6 million three type of grid, respectively ${ }^{[11]}$. The results showed that there were some differences between the rough grid and the medium grid, but the gap was small between the medium grid and fine grid. Therefore, the follow-up calculation was carried out with the 1.5 million medium meshes. Fig. 3 shows the jet pump simulation area. 


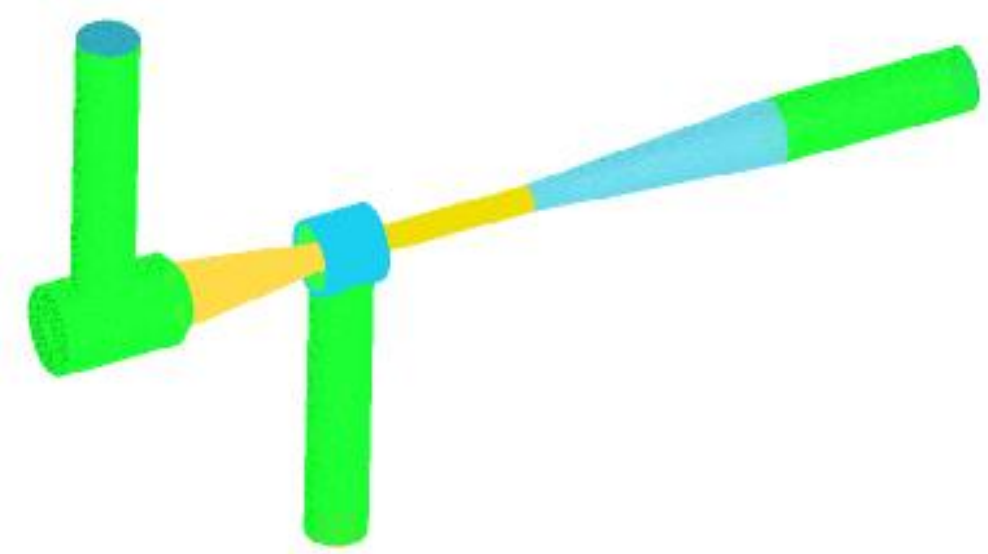

Fig. 3 Jet pump simulation area

\section{Model contrast}

The main feature of nozzle adjustable jet pump is that the nozzle could have different opening degrees, which can achieve the combination of different flow ratio and pressure ratio. Adjusting the opening and outlet pressure of jet pump, changing turbulence model and wall surface function, then doing simulation respectively. The opening of jet pump were $50 \%, 60 \%, 70 \%, 75 \%$ and $90 \%$, the outlet pressure were $1.4 \mathrm{MPa}, 2.0 \mathrm{MPa}, 2.5 \mathrm{MPa}, 3.0 \mathrm{MPa}, 3.5 \mathrm{MPa}$ and $4.0 \mathrm{MPa}$, respectively.

Fig. 4 compared the simulation values of the pressure and efficiency of the jet pump under five open degrees with the test value. It can be seen that the influence of turbulence model on the simulation results was obvious, while the wall function had less influence. The RNGKE model was significantly different from other models in the performance prediction of the internal flow field of the jet pump, the error was larger compared with the test value, so it's not suitable for the simulation of the nozzle adjustable jet pump. The results of SKE and RSM were similar to the experimental results, but existed deviations. The simulated value of RKE is highly coincided with the experimental value, and the trend of variation was similar, the error was small.The coincidence degree of SKESWF and SKENWF model curves was higher. It showed that the influence of wall function on simulation results can be neglected under the SKE model. The RSM model was also not sensitive to the wall function. As far as the RKE model is concerned, the NWF was closer to the experimental value than the SWF simulation results. Owing to the small internal size of the adjustable jet pump, the near wall is always in an unstable state of fluid separation and reattachment. Compared with the Standard wall function, the Non-Equilibrium wall function takes into account the influence of the pressure gradient near the wall, that is closer to the actual situation inside the nozzle adjustable jet pump, closer to test results. Therefore, the combination of RKE model and NWF wall function can predict the internal flow field of nozzle adjustable jet pump better.

It can also be seen from figure 4 that, with the increase of the pressure ratio, the jet pump efficiency at different opening first increased and then decreased, and the pressure ratio at the optimum operating point increased as well.

Fig. 5 is the jet pump efficiency error comparison curve, the error $e$ is defined by Eq. 5 .

$$
e=1-\frac{\text { simulate value }}{\text { experimential value }} \text {. }
$$

As can be seen from Figure 5, the RKE model was more suitable for the annular jet flow at large Reynolds numbers. And as the opening increases, the error between the 8 combinations and experimental values also decreased gradually. 

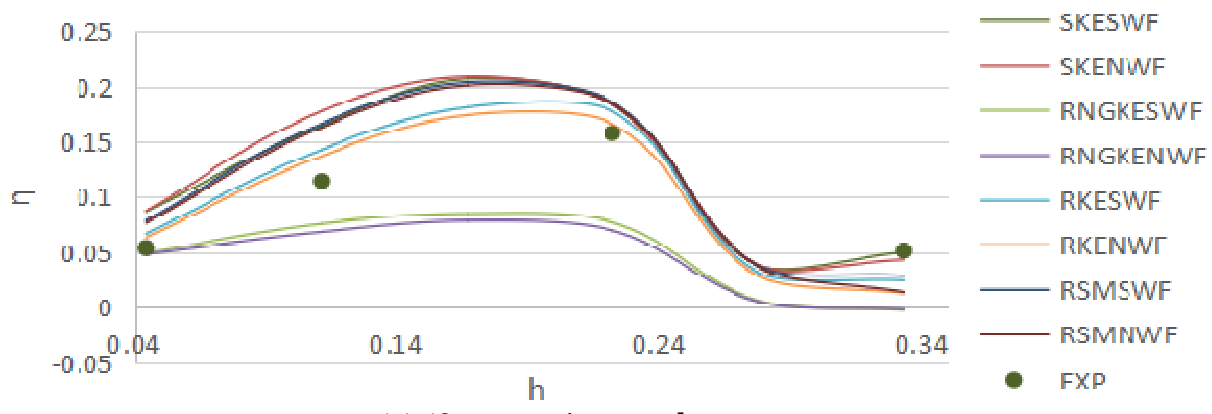

(a) $50 \%$ opening $h-\eta$ contrast

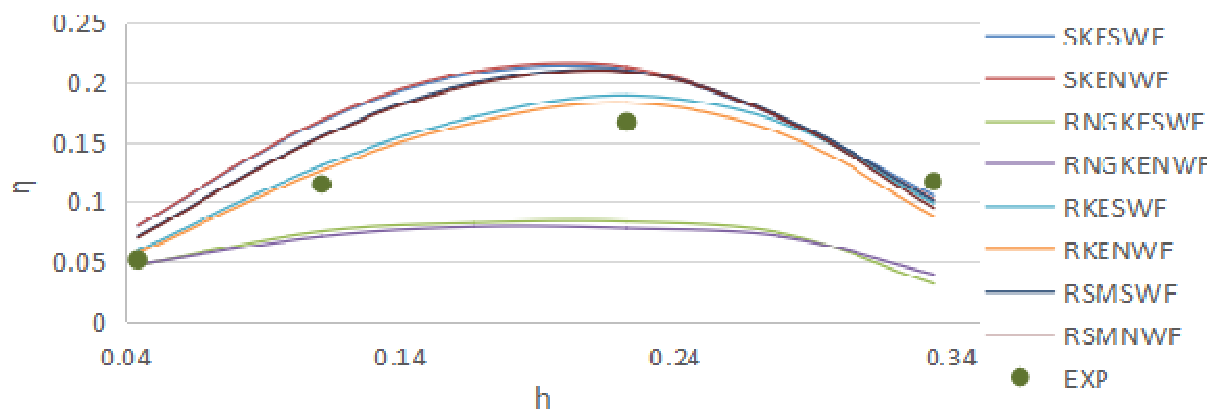

(b) $60 \%$ opening $h-\eta$ contrast
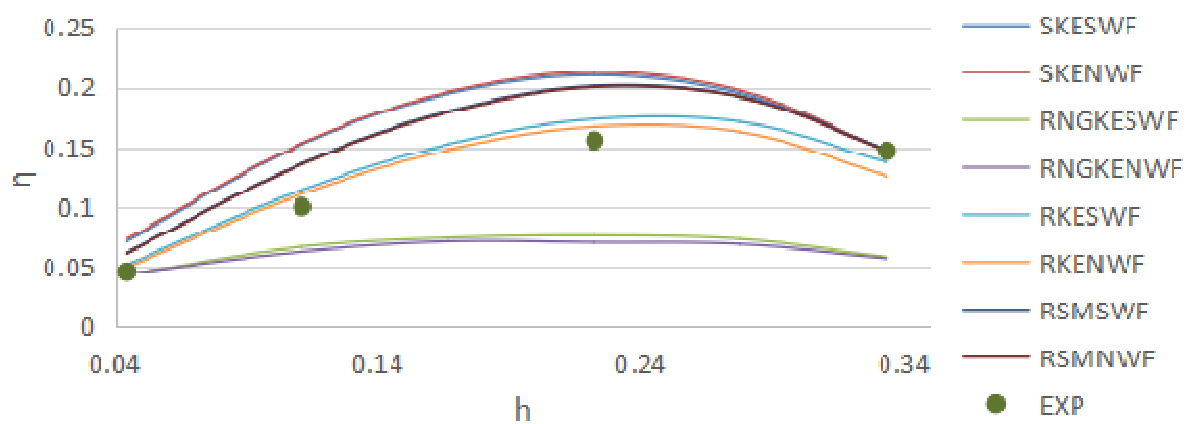

(c) $70 \%$ opening $h-\eta$ contrast

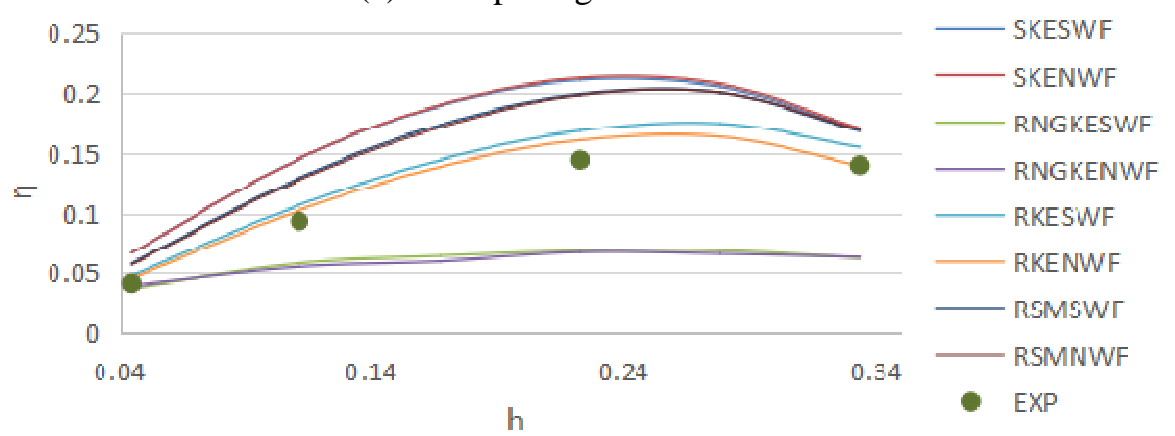

(d) $75 \%$ opening $h-\eta$ contrast

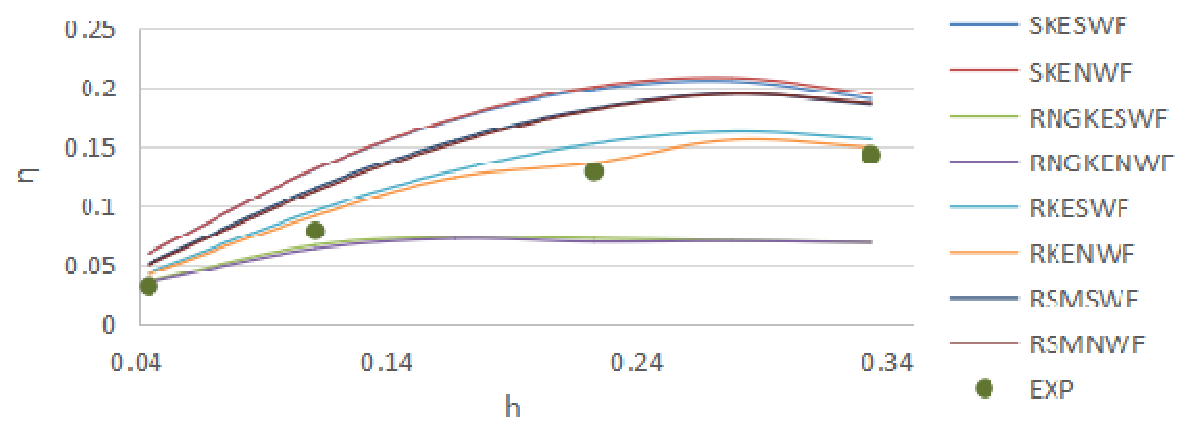

(e) $90 \%$ opening $h-\eta$ contrast

Fig. 4 Comparison of experimental and simulated values of jet pumps with different degrees of opening 

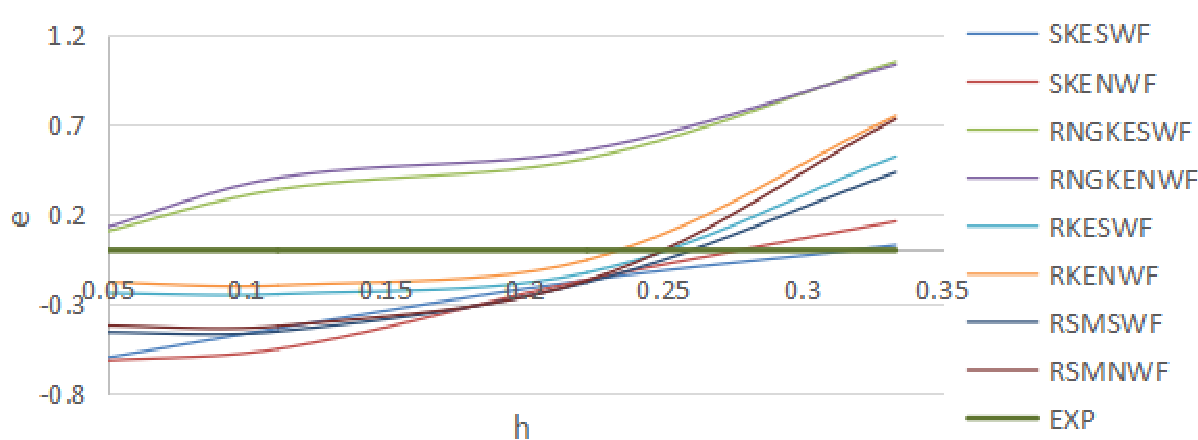

(a) $50 \%$ opening

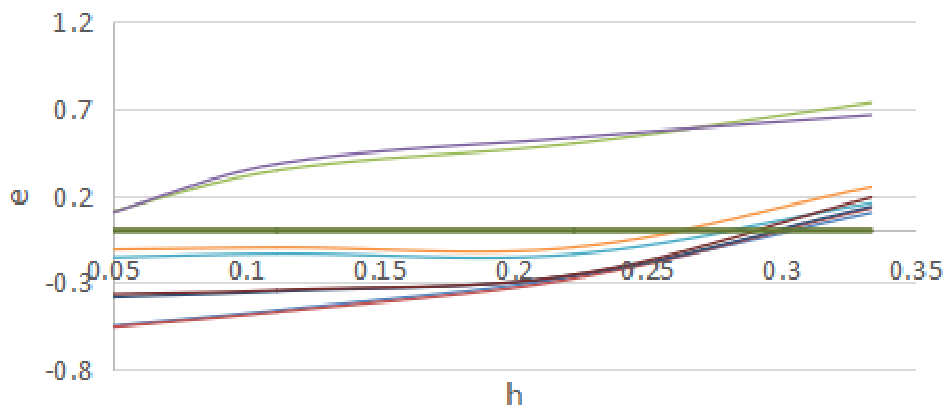

SKESWF

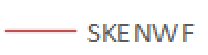

RNGKESWF

- RNGKENWF

RKESWF

- RKENWF

- RSMSWF

- RSMNWF

(b) $60 \%$ opening

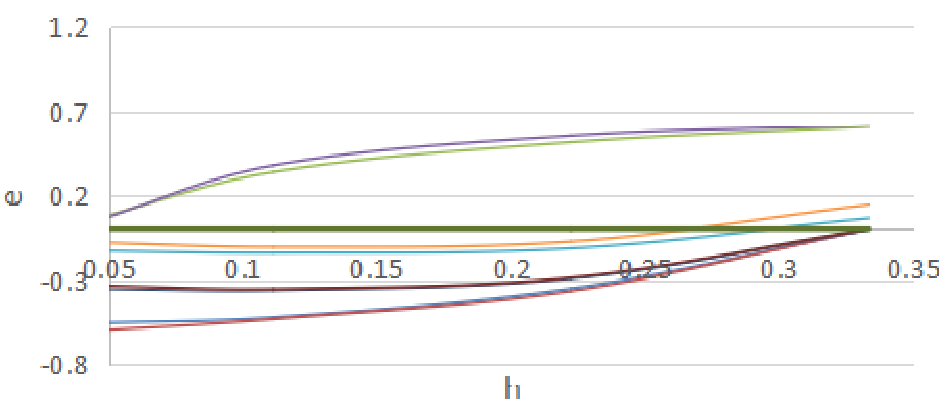

EXP

(c) $70 \%$ opening
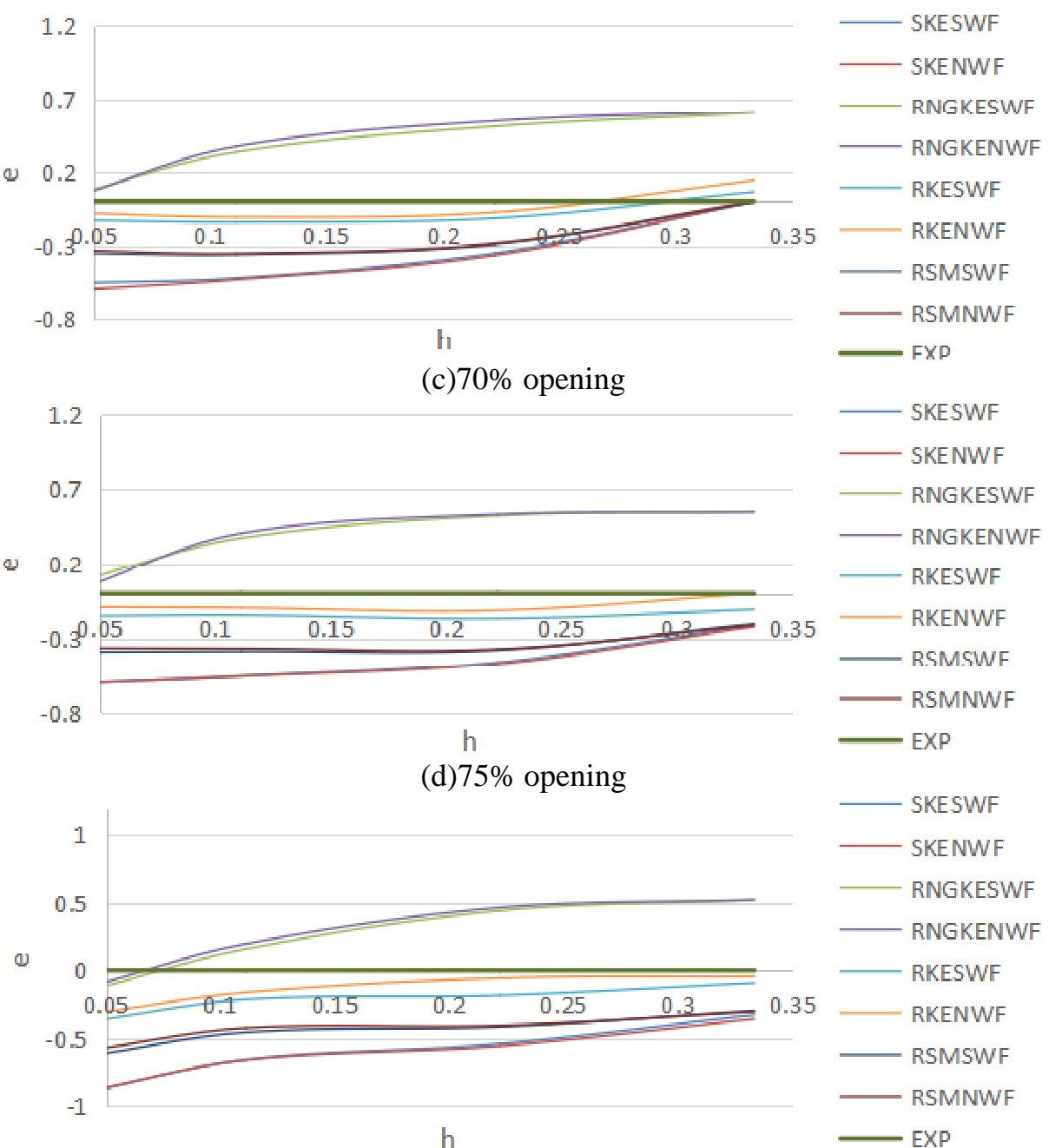

(e) $90 \%$ opening

Fig. 5 Comparison error of jet pump efficiency under different opening

In order to thoroughly compare the differences between different models, took the example of $50 \%$ opening and export pressure 3.0 MPa, Fig. 6 shows the turbulent kinetic energy nephograms of the jet 
pump shaft surfaces of the four models. It can be seen that the SKE, RKE and RSM models predicted similar trends in turbulent kinetic energy distributions, the working fluid was fully mixed with the absorbed liquid in the throat and were pressurized down at the diffusion tube. The simulation results of RNGKE model didn't have evident jet core and energy exchange transfer region, the differences were obvious. Calculated by the RKE model of turbulent kinetic energy region was the largest, most widely, and has been extended to the end of the diffusion tube, indicated that the working fluid and the intake fluid mixed fully.

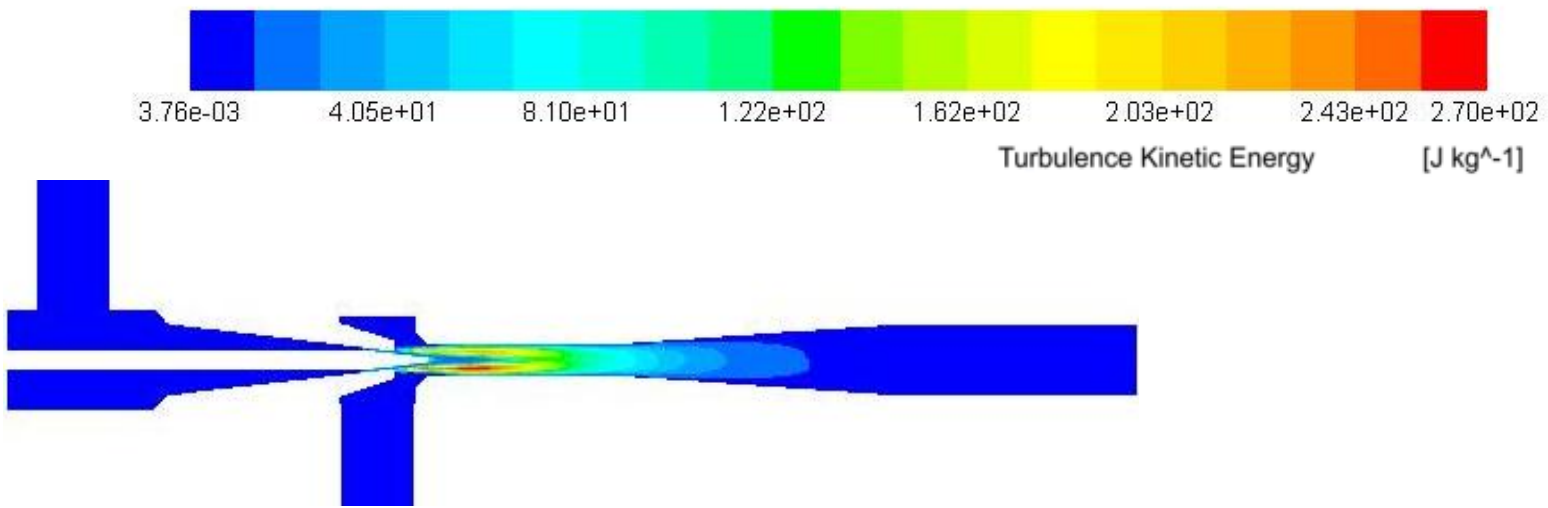

(a)SKENWF model turbulent kinetic energy nephograms

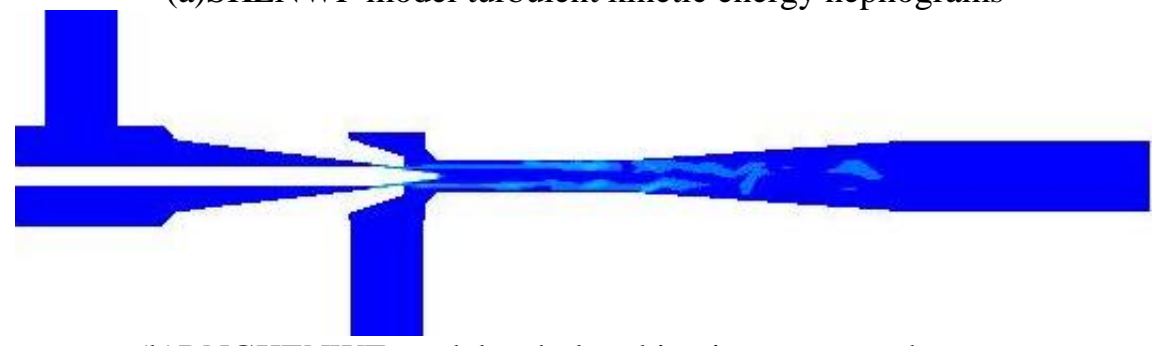

(b)RNGKENWF model turbulent kinetic energy nephograms

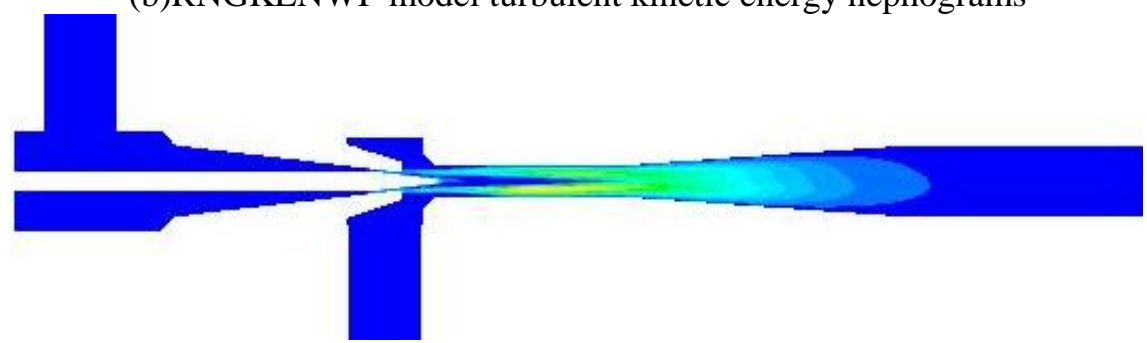

(c)RKENWF model turbulent kinetic energy nephograms

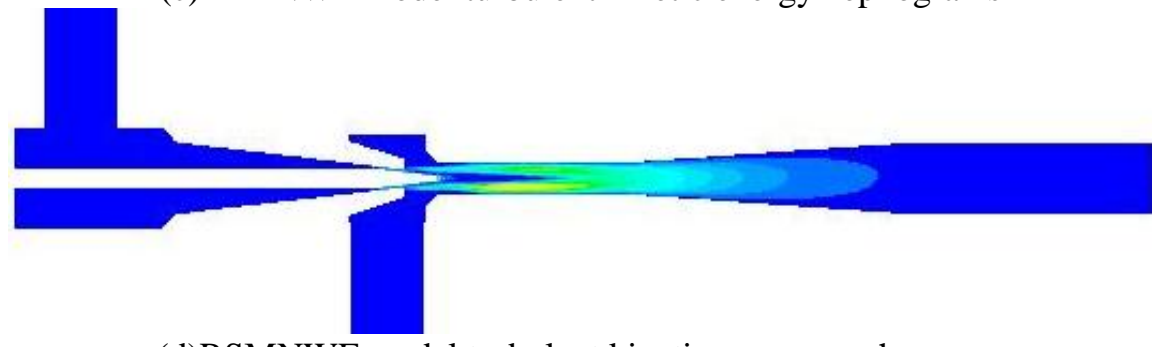

(d)RSMNWF model turbulent kinetic energy nephograms

Fig. 6 The turbulent kinetic energy nephograms of the jet pump shaft surfaces of the four models

In summary, it is advisable to use the Realizable model to simulate the adjustable jet pump of water injection nozzle for offshore oil field water injection. It can obtain more accurate variation trend of the internal performance of the jet pump, which is in good agreement with the experimental results. Compared with the Standard wall function, the Non-Equilibrium wall function is improved in solving the average velocity, considering the influence of the pressure gradient, it is more suitable for the simulation of the nozzle adjustable jet pump and can obtain higher precision. 


\section{Conclusions}

(1)The error of simulation results and test results decreased with the increase of opening and pressure ratio, and the pressure ratio of the optimum operating point increased with the increase of opening degree.

(2)The simulation values of SKE, RKE and RSM models are similar to the experimental values of the jet pumps with adjustable nozzles, and the trends are similar. But the result of RNGKE model is quite different from the experimental results, it is not applicable to the nozzle adjustable jet pump.

(3)The combination of Realizable model and Non-Equilibrium wall function can accurately predict the internal flow field of an adjustable jet pump for water injection in offshore oilfields, and the simulation results are accurate.

\section{References}

[1] Lu Hongqi. Theory and Application of Spray Technology[M]. Hubei Wuhan: Wuhan university press, 2004.

[2] Fan Ling, Liao Maolin, Liu Chao. Optimal Design of Water Injection System on Offshore Platform[J]. Oil-Gas field Surface Engineering, 2011, 30(9): 30-32.

[3] Lu Hongqi.Theory and Application of Jet Pump Technology[M]. Water Resources and Electric Power Press.1989.

[4] Long xinping, Guan yunsheng, Han ning. Numerical Simulation of the Performance of an Adjustable Jet Pump[J].Journal of Drainage and Irrigation Machinery Engineering, 2008, 26(6):1-5.

[5] Wang fujun. Computational Fluid Dynamics Analysis -- Principles and Applications of CFD Software[M]. Beijing, Tsinghua University Press, 2004.

[6] Yang xuelong, Long xinping, Xiao longzhong.Influence of Different Turbulence Models on Internal Flow Field Simulation of Jet Pump[J]. Journal of Drainage and Irrigation Machinery Engineering, 2013, 31(2):98-102.

[7] Zhou lingjiu, Yuan lingli. Comparison of Different Turbulence Simulation Methods for Internal Flow Calculation of Jet Pump[J].Journal of Drainage and Irrigation Machinery Engineering, 2013, 31(1):25-30.

[8] Hu xianghan, Kan ruiqing. Jet Pump Technology and Application [M].Haerbin, Heilongjiang Science and Technology Press, 1990.

[9] Wang jianjun, Li zhenlong, Ma haijiao. Review of Water Flooding Methods in Oilfield Development[J]. Inner Mongolia Petrochemical Industry, 2011(21): 25-27.

[10] Sokolov, Zenger, Huang qiuyun. Ejector[M]. Science Press Co.Ltd. 1977.

[11] Xiao L, Long X. Cavitating flow in annular jet pumps[J]. International Journal of Multiphase Flow,2015,71:116-132. 